\title{
To determine the prevalence of malnutrition in nursing and care homes in Walsall after six month re-screen using MUST
}

\author{
R. Ragubeer ${ }^{1}$ and E. Jerrams ${ }^{2}$ \\ ${ }^{1}$ Walsall Healthcare NHS Trust, Walsall WS2 9PS, UK and ${ }^{2}$ Nutricia Clinical Care, Wiltshire BA14 OXQ, UK
}

Malnutrition is common among older people in nursing, care and residential homes. It is frequently unrecognised and is often undertreated in the $\mathrm{UK}^{(1)}$. Malnutrition costs an estimated $£ 13$ billion in the $\mathrm{UK}^{(3)}$. It is recommended that all patients admitted to a nursing, care or residential home, be screened for malnutrition ${ }^{(2)}$. Despite this, there remains little information on the prevalence of malnutrition in this population. However, there is a large body of evidence demonstrating that if malnutrition is identified, it is treatable, resulting in both clinical improvements and economical savings. Malnutrition has a negative impact on a patient's health, and in turn this has cost implications for the NHS. Even a modest one percent decrease in the annual expenditure attributed to malnutrition would result in a saving in the region of $£ 130$ million per year ${ }^{(4)}$. Therefore identifying and appropriately treating malnutrition can avoid knock-on healthcare complications and inflated costs.

The audit aimed to establish whether the prevalence of malnutrition in the 6 nursing and care homes (5 nursing and 1 care home) initially screened using the 'Malnutrition Universal Screening Tool' (MUST) had remained the same, increased or decreased after a 6 month review. The study also aimed to determine how many residents were appropriately prescribed oral nutritional supplements (ONS), and by whom. The initial screening took place from January 2010 to May 2010.

Results from the initial screening showed that of the 249 residents (mean age $84 ; 73 \%$ female), $58 \%$ (144 residents) were screened as being low risk, $19 \%$ (46 residents) were screened as medium risk and $23 \%$ (58 residents) were screened as high risk. The mean BMI for each risk group was as follows: low risk $\left(26.0 \mathrm{~kg} / \mathrm{m}^{2}\right)$, medium risk $\left(21.4 \mathrm{~kg} / \mathrm{m}^{2}\right)$ and high risk $\left(17.8 \mathrm{~kg} / \mathrm{m}^{2}\right)$. (Please note: $1 \mathrm{resident}$ was admitted to hospital and no previous data available as she was a new resident). The results also showed that only $13 \%$ (including $~ 7.0 \%$ at high risk of malnutrition) of residents in the care homes taking supplements were being monitored and reviewed by a dietitian. 10 low risk and 8 medium risk residents had been prescribed ONS, either when discharged from hospital and from the GP. These residents were not being monitored. $42 \%$ of residents were at risk of malnutrition according to 'MUST' (19\% medium risk, $23 \%$ high risk). Residents in the nursing and care homes studied were inappropriately prescribed ONS without further review, $4.0 \%$ of low risk residents on ONS prescribed by either the hospital or GP, $3.0 \%$ of medium risk residents on ONS prescribed by the GP.

Six months after the initial screening, the clinical community dietitian and Nutricia Nurse re-screened the 6 nursing and care homes ( 5 nursing and 1 care home). 246 residents (mean age 85; 72\% female) were re-screened for malnutrition using 'MUST' from August 2010 to March 2011. 63\% (154 residents) were screened as being low risk, $12 \%$ (29 residents) were screened as medium risk and $24 \%$ (58 residents) were screened as high risk. The mean BMI for each risk group was as follows: low risk $\left(25.2 \mathrm{~kg} / \mathrm{m}^{2}\right), \mathrm{medium}$ risk $\left(19.2 \mathrm{~kg} / \mathrm{m}^{2}\right)$ and high risk $\left(18.2 \mathrm{~kg} / \mathrm{m}^{2}\right)$. (Please note: data for 3 residents was not available at the time of screening; 4 residents were not screened as they were in hospital).

Table 1. Percentage of screened residents receiving supplements and the source of the prescription

\begin{tabular}{lrcr}
\hline & Low risk & Medium risk & High risk \\
\hline Hospital & $1(0.4 \%)$ & $0(0 \%)$ & $1(0.4 \%)$ \\
GP & $14(5.7 \%)$ & $4(1.6 \%)$ & $4(1.6 \%)$ \\
Dietitian & $3(1.2 \%)$ & $2(0.8 \%)$ & $24(9.8 \%)$ \\
\hline
\end{tabular}

From Table 1, 12\% (including 9.8\% at high risk of malnutrition) of residents taking supplements were being monitored and reviewed by a dietitian. 15 low risk and 4 medium risk residents had been prescribed ONS either when discharged from hospital and from the GP. These residents were not being monitored. 2 of the residents had improved from high risk to medium risk, and 3 of the residents had improved from high risk to low risk and were being monitored by the dietitian. After the 'MUST' screening, these 24 residents who were on ONS were able to stop. Malnutrition is common in the nursing and care homes studied: $36 \%$ of residents were at risk of malnutrition according to 'MUST' (12\% medium risk, 24\% high risk). Residents in the homes studied were inappropriately prescribed ONS without further review (Table 1): $6.1 \%$ of low risk residents on ONS prescribed by either the hospital or GP, $1.6 \%$ of medium risk residents on ONS prescribed by the GP.

The re-screening showed that malnutrition in the homes re-screened after six months seemed to have decreased (from $42 \%$ at first screening to $36 \%$ at re-screen after 6 months). Without continued staff training and resident screening using 'MUST', malnutrition would have been unrecognised and untreated. The study also showed that identifying and appropriately treating malnutrition could potentially reduce health care costs.

1. Elia M (2003) The 'MUST' report. Nutritional screening for adults: a multidisciplinary responsibility. Redditch, UK, BAPEN.

2. NICE Guideline (2006) Nutrition Support in Adults. Clinical Guideline 32.

3. www.bapen.org.uk

4. Collins P (2011) Identifying Malnutrition in the Community. CN Focus Vol.3 No.2. 